\title{
Um modelo proativo de antecipação de ações de times de resposta rápida baseado em análise preditiva
}

\author{
Fabio de Oliveira Dias ${ }^{1,2}$, Cristiano André da Costa ${ }^{1}$, Rodrigo da Rosa Righi ${ }^{1}$ \\ ${ }^{1}$ Programa de Pós-Graduação em Computação Aplicada (PIPCA) \\ Universidade do Vale do Rio do Sinos (UNISINOS) \\ ${ }^{2}$ Instituto Federal Sul-rio-grandense (IFSul) \\ fabiodsapucaia.ifsul.edu.br, \{rrrighi, cac\}@unisinos.br
}

\begin{abstract}
A Rapid Response Team intends to prevent deaths in patients who have clinical deterioration outside of intensive care units in hospitals environments. The Predictvs model seeks to anticipate actions of rapid response teams, through the analysis of vital signs of patients with the use of early warning scores. The scientific contribution of the presented model is that we could better predict possible collapse situations of patients, through the monitoring and analysis of vital signs. The Predictvs evaluation was made using scenarios and their analysis showed encouraging results.
\end{abstract}

Resumo. Um Time de Resposta Rápida busca prevenir mortes de pacientes que tenham piora clínica fora de ambientes de Unidades de Tratamento Intensivo em hospitais. $O$ modelo Predictvs busca antecipar ações dos times de resposta rápida, através da análise dos sinais vitais dos pacientes com o uso de escores de alerta precoce. A contribuição científica do modelo é dada em virtude da possibilidade de efetuar a predição de possíveis situações de colapso dos pacientes através do monitoramento e análise dos sinais vitais. A avaliação do Predictvs foi efetuada com a utilização de cenários e a sua análise apresentou resultados que motivam a continuidade da pesquisa.

\section{Introdução}

Em dezembro de 2004, o fundador e CEO do Institute for Healthcare Improvement (IHI), uma organização independente e sem fins lucrativos que busca promover melhorias nos cuidados médicos, Dr. Don Berwick, lançou um desafio na reunião anual da entidade, ocorrida em Orlando. Impaciente com o ritmo das mudanças relacionadas à segurança dos pacientes e com objetivos vagos da área da saúde, ele anunciou uma campanha para salvar 100 mil vidas nos hospitais nos próximos 18 meses [Watcher and Pronovost 2006]. Para atingir esse audacioso objetivo, foi proposta a adoção de seis práticas pelas instituições de saúde. Dentre essas práticas estava a sugestão de implantação de Times de Resposta Rápida (Rapid Response Teams, RRTs) nos hospitais. Passados os 18 meses, o IHI anunciou que o objetivo inicial havia sido ultrapassado, tendo sido salvas 122.300 vidas [Watcher and Pronovost 2006]. Posteriormente, uma extensão dessa campanha foi lançada, propondo salvar 5 milhões de vidas entre dezembro de 2006 e dezembro de 2008 [McCannon et al. 2007].

Um Time de Resposta Rápida consiste em uma equipe de profissionais de saúde liderada por um médico, com a finalidade de prevenir mortes de pacientes que tenham piora clínica fora de um ambiente preparado para atendê-los em uma eventual situação crítica (por exemplo, pacientes em enfermarias). Para tanto, é necessária a análise de 
sinais vitais dos pacientes, de forma a prevenir ou detectar um eventual colapso dos mesmos [Berwick et al. 2006].

A computação móvel e ubíqua tem propiciado o advento de soluções que permitem o monitoramento em tempo real de sinais advindos de sensores e o seu processamento por aplicações que podem executar ações de acordo com as condições encontradas. Esta característica possibilita o uso da computação móvel e ubíqua para o monitoramento de condições de saúde de pacientes, denominado de cuidados ubíquos (ubiquitous healtcare) [Gelogo et al. 2013]. Indo além, provê, inclusive, condições para que possa ser efetuada a predição de eventuais pioras clínicas e o acionamento de profissionais de saúde para prestar o adequado socorro, permitindo o salvamento de vidas.

Este trabalho se insere nestas condições, estando voltado para a antecipação de ações de times de resposta rápida baseado em análise preditiva, com o uso de escores de alerta precoce, propondo o modelo Predictvs. A Figura 1 apresenta o cenário em que o modelo proposto se encaixa, demonstrando a transmissão dos dados advindos dos sensores e o envio de sinais de alerta aos profissionais de saúde e ao Time de Resposta Rápida.

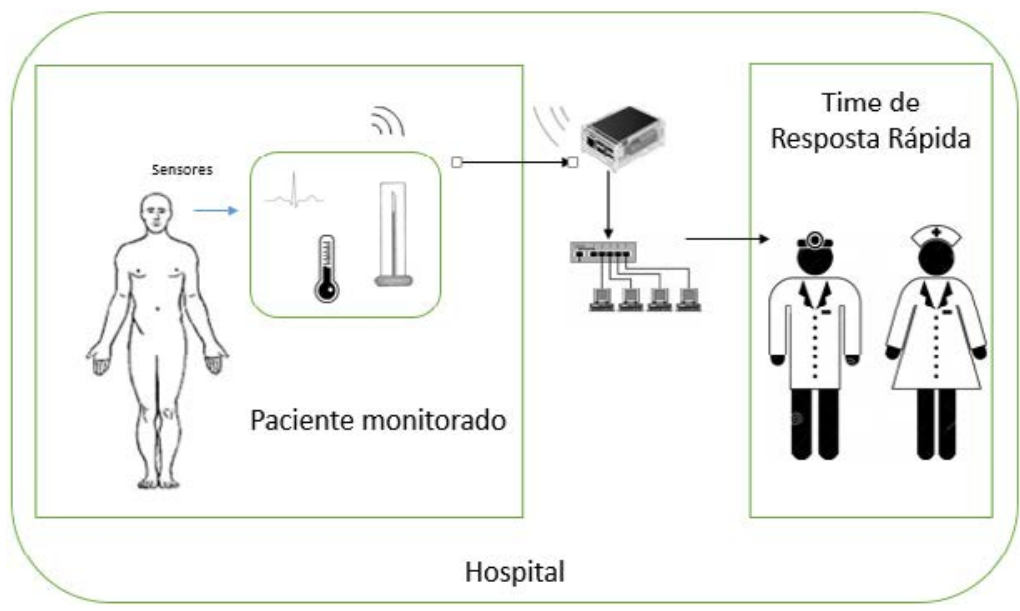

Figura 1. Cenário de utilização do modelo Predictvs

A principal contribuição científica do trabalho decorre da possibilidade de, através do monitoramento dos sinais vitais com o uso de escores de alerta precoce, prever possíveis situações de colapso dos pacientes e antecipar o acionamento dos times de resposta rápida. Não foram encontrados, durante as pesquisas de trabalhos relacionados, modelos com essa característica. Os trabalhos relacionados demonstraram que o uso de escores de alerta precoce são uma importante ferramenta utilizada pelos profissionais de saúde para efetuar a medição do estado clínico dos pacientes, mas as totalizações geralmente ocorrem de forma manual, em formulários de papel situados junto ao prontuário dos pacientes [Morgan et al. 1997; Kyriacos et al. 2014]. O modelo propõe a automatização da totalização da pontuação do escore de alerta precoce, possibilitando a antecipação do acionamento dos times de resposta rápida.

Este trabalho foi dividido em seis seções. Após a introdução, a seção 2 descreve, brevemente, a relação proposta entre escores de alerta precoce com os times de resposta rápida. A seção 3 descreve a arquitetura do modelo Predictvs. A seção 4 apresenta uma avaliação do modelo baseada em cenários. Trabalhos relacionados são apresentados na seção 5. Finalmente, as conclusões são apresentadas na seção 6. 


\section{Escores de Alerta Precoce}

Em 1997, Morgan, Williams e Wright [Morgan et al. 1997] foram os primeiros a desenvolver e publicar um escore de alerta precoce (EWS) no Reino Unido. Este EWS fazia referência a cinco parâmetros fisiológicos: frequência cardíaca, pressão arterial sistólica, frequência respiratória, temperatura e nível de consciência. Cada parâmetro possui uma gama de pontos de corte com gatilhos correspondentes, não para efetuar a predição, mas para servir como um sistema de rastreamento e gatilho para identificar os primeiros sinais de deterioração dos pacientes. Por exemplo, uma frequência cardíaca entre 111 e $129 \mathrm{bpm}$ (batimentos por minuto) recebe um escore de 2 pontos, indicando a necessidade de escalada para intervenção pela equipe médica.

Desde então, os sistemas de EWS foram modificados (sendo denominados MEWS, escore de alerta precoce modificado) e padronizados por todo o Reino Unido. Em adição aos cinco parâmetros fisiológicos citados, outros foram incorporados na maioria dos MEWS, tais como nível de saída da urina e oxigenação do sangue. Como não existe um MEWS ideal, a multiplicidade destes sistemas resultou em uma falta de consistência no reconhecimento e na resposta a deterioração clínica, tornando necessária a sua padronização. Em julho de 2012 foi implementado um escore de alerta precoce nacional (NEWS), com o monitoramento de seis parâmetros fisiológicos (frequência respiratória, saturação de oxigênio, temperatura, pressão arterial sistólica, frequência cardíaca e nível de consciência), porém a adoção deste não se tornou obrigatória nos hospitais [Kyriacos et al. 2014]. A Tabela 1 mostra um exemplo de tabela de escore de alerta precoce [Kyriacos et al. 2014].

\begin{tabular}{|c|c|c|c|c|c|c|c|}
\hline Sinal fisiológico/Escore & 3 & 2 & 1 & $\mathbf{0}$ & 1 & 2 & 3 \\
\hline Frequência respiratória/min & & $\leq 9$ & & $9-14$ & $15-20$ & $21-29$ & $\geq 30$ \\
\hline Saturação $\mathrm{O}_{2}$ & $<85$ & $85-89$ & $90-92$ & $\geq 93$ & & & \\
\hline Frequência cardíaca/min & & $\leq 40$ & $41-50$ & $51-100$ & $101-110$ & $111-129$ & $\geq 130$ \\
\hline Pressão sistólica & $\leq 70$ & $71-80$ & $81-100$ & 101-199 & & $\geq 200$ & \\
\hline Temperatura ${ }^{\circ} \mathrm{C}$ & & $\leq 35$ & & $35-38.4$ & & $\geq 38.5$ & \\
\hline $\begin{array}{c}\text { Estado neurológico } \\
\text { Escala de Coma Glasgow }\end{array}$ & & & & 15 & 14 & $13-9$ & $\leq 8$ \\
\hline $\begin{array}{c}\text { Estado neurológico } \\
\text { Escala AVPU }\end{array}$ & & & & Alerta & $\begin{array}{c}\text { Reage à } \\
\text { voz }\end{array}$ & $\begin{array}{c}\text { Reage à } \\
\text { dor }\end{array}$ & $\begin{array}{l}\text { Sem } \\
\text { resposta }\end{array}$ \\
\hline Urina $\mathrm{ml} / \mathrm{kg} /$ hora & $\begin{array}{c}0.5 \mathrm{ml} / \mathrm{kg} \\
/ 1 \text { hora }\end{array}$ & & $\begin{array}{c}\leq 1 \\
\mathrm{ml} / \mathrm{kg} / 1 \\
\text { hora }\end{array}$ & & $\begin{array}{c}\geq 3 \\
\mathrm{ml} / \mathrm{kg} / 1 \\
\text { hora }\end{array}$ & & \\
\hline
\end{tabular}

Tabela 1. Exemplo de escore de alerta precoce [Kyriacos et al. 2014]

A análise de sinais vitais de pacientes permite monitorar possíveis ocorrências para acionar os times de resposta rápida. As ocorrências normalmente são classificadas em duas chamadas: Código Amarelo para urgências e Código Azul para emergências PCR (Parada Cardiorrespiratória). Estudos comprovam a eficiência da abordagem, reduzindo a quantidade de óbitos nessas condições de forma significativa [Offner et al. 2007; Gonçalves et al. 2012]. Abaixo são descritos alguns exemplos de sinais de alerta que podem disparar as ações dos Times de Resposta Rápida:

bpm;

- Alteração súbita na frequência cardíaca, ficando abaixo de 40 ou acima de 130 
- Alteração súbita na pressão arterial sistólica, ficando menor que $90 \mathrm{mmHg}$ (milímetros de mercúrio);

- Alteração súbita na frequência respiratória, estando abaixo de 8 ou acima de 28 ipm (inspirações por minuto), ou com comprometimento de via aérea;

- Alteração súbita de nível de consciência [Offner et al. 2007].

\section{Modelo Predictvs}

O modelo proposto permite o acionamento do Time de Resposta Rápida do hospital em virtude da predição da deterioração do estado clínico do paciente através do uso de escores de alerta precoce. Conforme [Bailey et al 2013], o tempo é um fator essencial para intervenções médicas em situações de urgência e emergência, de forma a prevenir o agravamento do estado clínico dos pacientes. Partindo dessa premissa, o modelo Predictvs foi planejado para ter uma arquitetura simples, de forma a dispender o tempo mínimo no tratamento dos sinais fisiológicos, porém eficiente, provendo os alertas necessários e adequados para os times de resposta rápida. A Figura 2 exibe a arquitetura do modelo Predictvs.

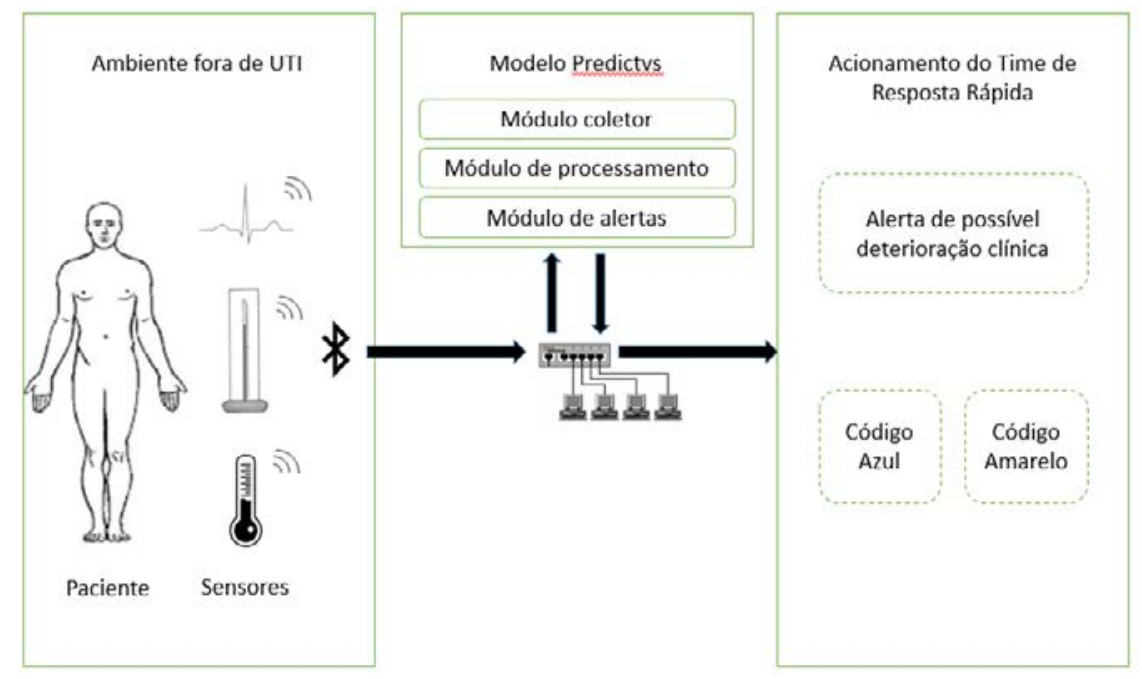

Figura 2. Arquitetura do modelo Predictvs

Inicialmente, o perfil do paciente deve ser informado ao sistema, bem como o tipo de escore de alerta precoce que será utilizado. O modelo permite o uso de múltiplos escores de alerta precoce, que devem ser cadastrados previamente pelo médico responsável. Os thresholds dos sinais vitais presentes no escore de alerta precoce atribuído pelo médico também podem ser personalizados, de forma a refletir eventuais peculiaridades no estado clínico do paciente, permitindo uma predição mais apurada.

Sensores acoplados aos pacientes efetuam a leitura de seus sinais fisiológicos (tais como frequência cardíaca, frequência respiratória, pressão arterial sistólica e temperatura) e os transmitem para um módulo coletor via Bluetooth. O módulo coletor consiste em um dispositivo, conectado à rede local da instituição, que possui a finalidade de transmitir os dados lidos ao módulo de processamento do modelo. O modelo suporta múltiplos módulos coletores, provendo escalabilidade e redundância ao sistema.

O módulo de processamento, através da função de análise da leitura efetuada, verifica se o sinal fisiológico foi detectado corretamente ou se houve alguma discrepância, 
que leva ao descarte do dado e à solicitação de nova leitura. Em seguida, a função de pontuação do escore de alerta precoce faz o cálculo da pontuação dos sinais vitais lidos, com base no escore de alerta precoce configurado pelo médico. Escores de até 3 pontos geram alertas do tipo warning para o Time de Resposta Rápida, indicando possível necessidade de intervenção; escores acima deste valor geram alertas do tipo critical contendo um Código Azul (se houve parada cardiorrespiratória) ou um Código Amarelo (se houve outro tipo de colapso), obrigando o acionamento da equipe. A Figura 3 exibe a arquitetura do módulo de processamento.

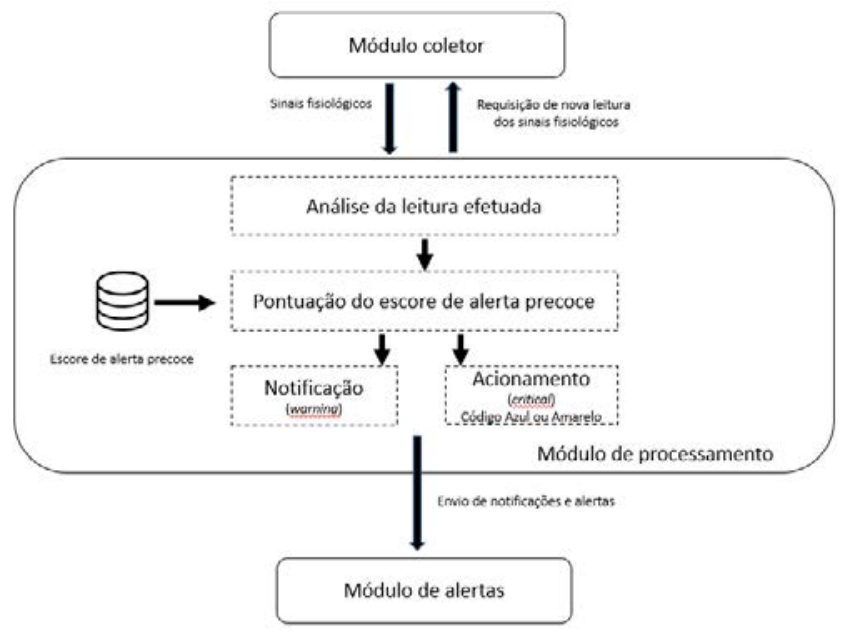

Figura 3. Módulo de processamento

O módulo de alertas é responsável por transmitir estes avisos, através da função envio de alertas. De forma sucinta, o modelo prevê que os profissionais de saúde devem assinar uma fila de tópicos de mensagens para receber os alertas do Predictvs. Assim, o módulo de alertas efetua a transmissão da mensagem e, de acordo com as condições do paciente, os integrantes do time de resposta rápida recebem as notificações e os acionamentos.

\section{Avaliação}

A utilização de cenários tem sido empregada pela comunidade científica para a avaliação de aplicações ubíquas [Satyanarayanan, 2011; Rocha, 2015]. Desta forma, foi construído um cenário para a avaliação do modelo apresentado na seção anterior. $\mathrm{O}$ cenário demonstra a atuação do Predictvs na predição da deterioração clínica dos pacientes, com base nos sinais vitais coletados. O cenário utilizado foi:

"Júlia, por requisição de seu médico, em virtude de seu histórico de problemas cardíacos, vai ao centro clínico de seu hospital para que seja realizado um ecodopplercardiograma bidimensional com mapeamento de fluxo em cores, um importante exame para pacientes que possuem doenças relacionadas ao coração. Ao chegar, Júlia recebe uma série de sensores, que são acoplados ao seu corpo e que se comunicam com um dispositivo coletor Raspberry Pi conectado à rede local do hospital. Tais sensores efetuam, periodicamente, a leitura de sua frequência cardíaca, frequência respiratória, pressão arterial sistólica e temperatura e repassam os dados ao dispositivo coletor, que os retransmite ao módulo de processamento do sistema Predictvs. Júlia realiza o exame e é deixada em repouso na enfermaria. Em determinado momento, o Predictvs detecta que seus sinais vitais começam a se deteriorar, com seu escore de alerta 
precoce atingindo a pontuação 3. Com base nisto, o módulo de alertas do sistema envia um alerta do tipo warning ao Time de Resposta Rápida, indicando tal situação. No entanto, a deterioração de seus sinais vitais torna-se ainda mais aguda (com a pontuação de seu escore de alerta precoce chegando a 7) e o sistema Predictvs emite um novo alerta, agora do tipo critical, contendo um Código Azul, acionando os integrantes do Time de Resposta Rápida do hospital. Assim, os devidos cuidados são aplicados à paciente."

Para que a avaliação do cenário exposto pudesse ser efetuada, um protótipo baseado em Python, com uma interface web JavaScript, foi desenvolvido. O módulo de processamento recebe os sinais vitais através do uso das filas de tópicos do middleware RabbitMQ. Após, efetua a análise dos sinais e atribui um escore ao paciente. Quando necessário, o módulo de alertas envia mensagens, também através do uso das filas de tópicos do middleware RabbitMQ, ao Time de Resposta Rápida do hospital, avisando-o sobre possível colapso ou acionando-o em caso de ocorrência de um Código Azul ou Código Amarelo.

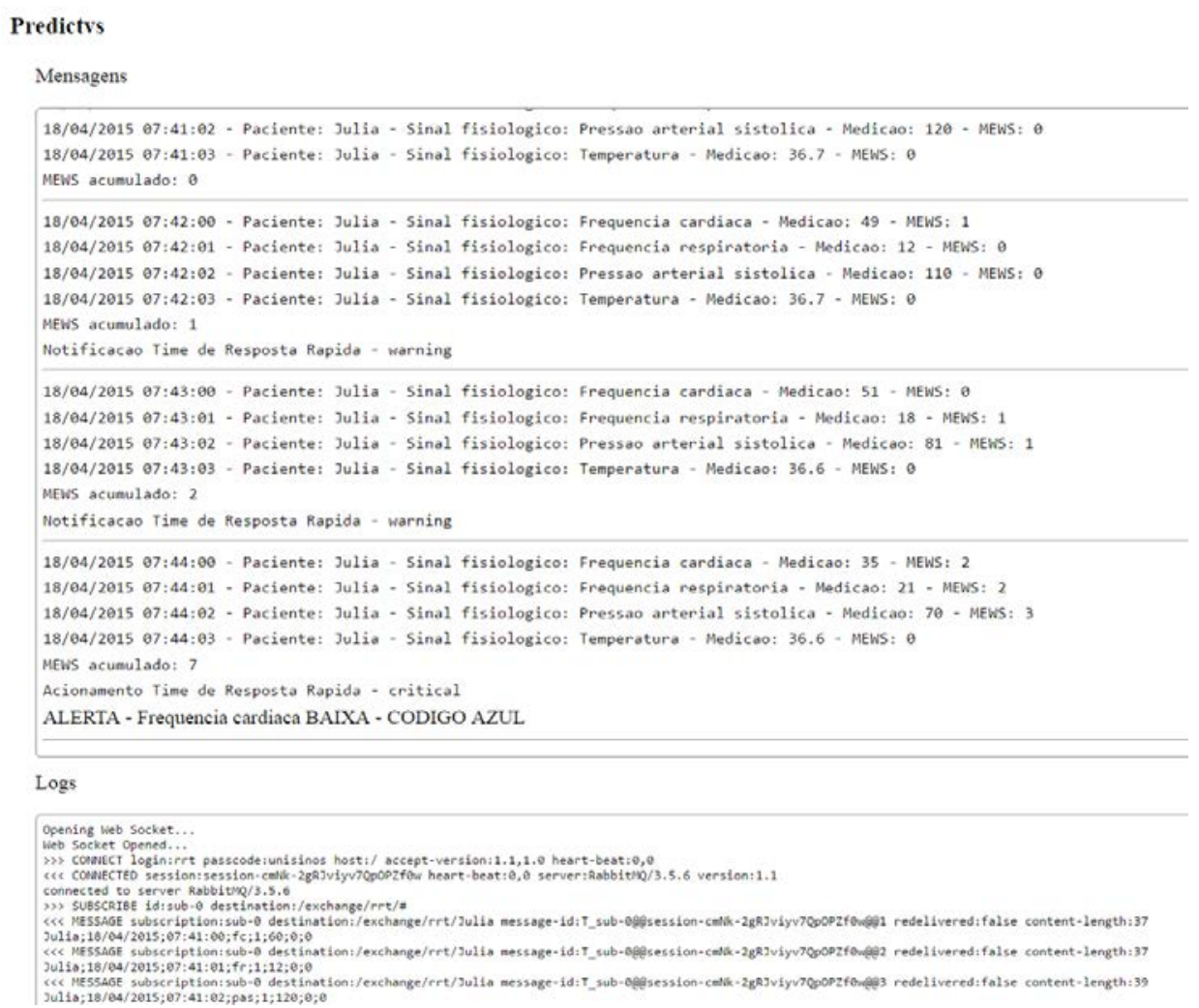

Figura 4. Protótipo do Predictvs em ação

A utilização do Predictvs requer o cadastro de algumas informações relacionadas ao paciente. Basicamente deve ser informada a identificação do paciente e o perfil de escore de alerta precoce a ser utilizado. O Predictvs é extremamente customizável. Além do escore de alerta precoce ser adaptável, permitindo a criação e utilização de múltiplos escores de alerta precoce modificados (MEWS), os profissionais de saúde podem atribuir os thresholds que julgarem apropriados em cada item do escore. A Figura 4 apresenta a tela do módulo de processamento em ação, demonstrando o escore MEWS calculado a cada ciclo de leitura dos sensores, bem como as notificações e o acionamento do time de resposta rápida. Os parâmetros de escore de alerta precoce utilizados são os descritos na Tabela 1. 
Assim que foram acoplados em seu corpo, os sensores passaram a reportar, via Bluetooth, os dados de frequência cardíaca, frequência respiratória, pressão arterial sistólica e temperatura de Júlia. Em determinado momento, logo após Júlia ter realizado o exame e ter sido colocada em repouso, sua frequência cardíaca estava em $51 \mathrm{bpm}$, sua frequência respiratória estava em 18 ipm e sua pressão arterial sistólica estava em 81 mmHg. O módulo de pontuação do Predictvs atribuiu 2 pontos a essa condição clínica, o que disparou um alerta de possível colapso ao Time de Resposta Rápida. Entretanto, poucos minutos depois, as condições clínicas de Júlia deterioraram-se ainda mais: sua frequência cardíaca baixou para $35 \mathrm{bpm}$, sua frequência respiratória subiu para $21 \mathrm{ipm}$ e sua pressão arterial sistólica baixou para $70 \mathrm{mmHg}$. Neste caso, a condição clínica de Júlia recebeu 7 pontos do módulo de pontuação, o que gerou o acionamento do Time de Resposta Rápida do hospital com um Código Azul. Assim, Júlia recebeu os devidos cuidados médicos, sendo transferida para a Unidade de Tratamento Intensivo do hospital e teve a sua vida salva.

\section{Trabalhos relacionados}

Foram considerados cinco trabalhos que possuem características semelhantes ao modelo proposto. O trabalho de [Kirkland et al. 2013] propõe o desenvolvimento de uma ferramenta para predição da deterioração clínica de pacientes não monitorados durante seu tempo de permanência em um hospital, utilizando, para tanto, variáveis procedentes de análise de regressão $\mathrm{A}$ análise final de regressão multivariada demonstrou que as variáveis Escala de Braden, frequência respiratória, saturação de oxigênio arterial e índice de choque hipovolêmico foram associadas de forma independente aos eventos. Frequência cardíaca e temperatura foram eliminadas do modelo final, apesar da primeira já estar representada através do índice de choque hipovolêmico.

O artigo de [Kyriacos et al. 2014] procura desenvolver e validar, por consenso entre especialistas da área de saúde, a construção e o conteúdo de uma tabela de observações para enfermeiros, incorporando um escore de alerta precoce modificado (MEWS) para o monitoramento de pacientes em um hospital público na África do Sul. O MEWS se baseou em sete parâmetros fisiológicos (frequência respiratória, frequência cardíaca, pressão arterial sistólica, temperatura, nível de consciência, saturação de oxigênio - $\mathrm{SaO} 2$ e nível da saída de urina) e em uma tabela de observações.

[Brady et al. 2013] apresentam um sistema que busca identificar e mitigar riscos de colapsos de pacientes utilizando princípios de organizações de alta confiabilidade, como os empregados em usinas nucleares e empresas de aviação comercial. Estas organizações lidam com riscos constantes e mantêm recordes de segurança exemplares. Foram analisados eventos adversos sérios mais recentes e as transferências para a UTI. Cinco fatores de risco foram associados com cada evento: familiares, terapias de alto risco, presença de alto escore de alerta precoce (EWS), problemas intestinais e de comunicação. Usando o modelo como base para melhorias, uma intervenção foi desenvolvida e testada para identificar de forma proativa o risco de deterioração do estado clínico dos pacientes. Após a intervenção, as taxas de transferência para a UTI em virtude de eventos de falha de contextos não reconhecidos reduziu de forma significativa de 4,4 para 2,4 durante o período do estudo.

O objetivo do trabalho de [Badriyah et al. 2013] é comparar a performance de um escore de alerta precoce nacional (NEWS), gerado por humanos e otimizado por tentativa 
e erro, com um escore de alerta precoce baseado em árvore de decisão (DTEWS), gerado totalmente por meios computacionais. Os autores avaliaram a habilidade do DTEWS de discriminar pacientes com risco de parada cardíaca, admissão imprevista em UTI ou morte, cada um dentro de 24 horas a partir de uma dada observação de sinais vitais. Foram comparadas as performances de DTEWS e NEWS utilizando a área sob a curva característica de operação do receptor (curva ROC), tal como no primeiro artigo estudado. Os autores concluem o trabalho afirmando que a técnica de árvore de decisão valida de forma independente o padrão NEWS. A abordagem por árvore de decisão provê rapidamente um escore de alerta precoce quase idêntico ao NEWS.

O artigo de [Bailey et al. 2013] mostra um ensaio de predição em tempo real com pacientes em enfermarias. O objetivo central do estudo era validar um algoritmo preditivo da piora clínica em pacientes da ala médica geral e conduzir um teste de alertas em tempo real baseados neste algoritmo. Esses alertas em tempo real foram gerados por um algoritmo projetado para prever a necessidade de transferência para UTI usando dados eletronicamente disponíveis. Os alertas eram enviados ao enfermeiro chefe da ala. Para tanto, foi desenvolvida uma ferramenta de predição em tempo real, utilizando o particionamento recursivo da análise da árvore de regressão para identificar iminentes infecções. Essa implementação com base na ferramenta resultou em aumento de intervenções antecipadas, incluindo escalada de antibióticos, fluídos intravenosos, oxigenoterapia e diagnósticos em pacientes identificados como de risco.

Tabela 2. Comparação de características entre os trabalhos relacionados

\begin{tabular}{|c|c|c|c|c|c|c|}
\hline \multirow{2}{*}{\multicolumn{2}{|c|}{ Características }} & \multicolumn{5}{|c|}{ Trabalhos } \\
\hline & & $\begin{array}{c}\text { Kirkland et al., } \\
2013\end{array}$ & $\begin{array}{c}\text { Kyriacos et al., } \\
2014\end{array}$ & $\begin{array}{l}\text { Brady et al., } \\
2013\end{array}$ & $\begin{array}{l}\text { Badriyah et al., } \\
2013\end{array}$ & $\begin{array}{c}\text { Bailey et al., } \\
2013\end{array}$ \\
\hline \multicolumn{2}{|c|}{ Técnica utilizada } & $\begin{array}{c}\text { Escala de } \\
\text { Braden } \\
\text { Semi-Markov }\end{array}$ & $\begin{array}{l}\text { Sistema de } \\
\text { escores } \\
\text { Thresholds }\end{array}$ & $\begin{array}{l}\text { Algoritmo de } \\
\text { ciência de } \\
\text { situação }\end{array}$ & $\begin{array}{l}\text { Árvore de } \\
\text { decisão }\end{array}$ & $\begin{array}{l}\text { Regressão } \\
\text { logística }\end{array}$ \\
\hline \multirow{5}{*}{ 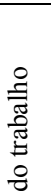 } & Pressão sistólica & $\cdot$ & $\cdot$ & & $\bullet$ & $\bullet$ \\
\hline & Pressão diastólica & $\cdot$ & & & $\cdot$ & $\cdot$ \\
\hline & Frequência cardíaca & $\cdot$ & $\cdot$ & & $\bullet$ & - \\
\hline & $\begin{array}{l}\text { Índice de choque } \\
\text { hipovolêmico }\end{array}$ & $\cdot$ & & & & $\bullet$ \\
\hline & Frequência respiratória & $\cdot$ & $\cdot$ & & $\bullet$ & $\cdot$ \\
\hline \multirow{7}{*}{ 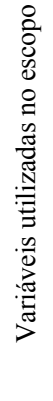 } & Frequência do pulso & & & & $\cdot$ & \\
\hline & Temperatura & $\cdot$ & $\cdot$ & & $\cdot$ & \\
\hline & Saturação $\mathrm{O}_{2}$ & $\cdot$ & $\cdot$ & & $\bullet$ & $\cdot$ \\
\hline & Nível de consciência & & $\cdot$ & & $\cdot$ & \\
\hline & $\begin{array}{l}\text { Cinco fatores de risco: } \\
\text { familiares, terapias de alto } \\
\text { risco, presença de alto } \\
\text { EWS, problemas } \\
\text { intestinais } \\
\text { comunicação. }\end{array}$ & & & $\cdot$ & & \\
\hline & Escala de Braden & $\cdot$ & & & & \\
\hline & Medição do nível de urina & & $\cdot$ & & & \\
\hline \multicolumn{2}{|c|}{$\begin{array}{l}\text { Suporte computacional descrito } \\
\text { no trabalho }\end{array}$} & Não & Não & Sim & Sim & Sim \\
\hline \multicolumn{2}{|c|}{ Faz uso de ciência de situação } & Não & Não & Sim & Não & Não \\
\hline \multicolumn{2}{|c|}{ Padrões utilizados } & $\begin{array}{l}\text { Baseado em } \\
\text { EWS }\end{array}$ & MEWS & EWS e PEWS & $\begin{array}{l}\text { NEWS e } \\
\text { DTEWS }\end{array}$ & $\begin{array}{l}\text { Atribuição de } \\
\text { escores } \\
\text { baseados em } \\
\text { EWS } \\
\end{array}$ \\
\hline \multicolumn{2}{|c|}{ Elaboração de protótipo } & Não & Não & Não & Não & Sim \\
\hline \multicolumn{2}{|c|}{$\begin{array}{l}\text { Modelo proposto relaciona-se } \\
\text { com Times de Resposta Rápida }\end{array}$} & Não & Não & Não & Não & Não \\
\hline
\end{tabular}


A Tabela 2 apresenta um comparativo entre os trabalhos relacionados. O modelo proposto neste trabalho procura preencher as lacunas encontradas, permitindo o uso de praticamente qualquer medição de sinais fisiológicos que possa ser efetuada com o uso de sensores, sendo compatível com múltiplos escores de alerta precoce e propiciando, como explanado nas seções anteriores, o acionamento de times de resposta rápida.

\section{Conclusão}

O presente trabalho apresentou o modelo Predictvs, que permite antecipar ações dos times de resposta rápida dos hospitais através do monitoramento dos sinais vitais dos pacientes utilizando escores de alerta precoce. O protótipo apresentado mostrou, no cenário efetuado, um competente instrumento na tarefa de efetuar a análise de sinais vitais advindos de sensores dos pacientes, de forma a prevenir ou detectar um eventual colapso dos mesmos e, nos casos pertinentes, enviar os devidos alertas ao Time de Resposta Rápida.

Como trabalhos futuros, pretende-se desenvolver um protótipo que possa ser aplicado em diferentes situações reais. Para tanto, planeja-se a realização de estudo de caso em ambiente monitorado, de forma a analisar e comprovar a eficiência da modelo.

\section{Agradecimentos}

Os autores gostariam de agradecer ao CNPq pelo apoio a esta pesquisa.

\section{Referências}

Ameyed D., Miraoui M., Tadj C. (2015). "A Survey of Prediction Approach in Pervasive Computing”. International Journal of Scientific \& Engineering Research.

Berwick D. et al. (2006). "The 100,000 Lives Campaign: Setting a goal and a deadline for improving health care quality". The Journal of the American Medical Association, JAMA, 295:324-327.

Bailey, T. C. et al. (2013). "A Trial of a Real-Time Alert for Clinical Deterioration in Patients Hospitalized on General Medical Wards". Society of Hospital Medicine. DOI 10.1002/jhm.2009.

Badriyah, T. et al. (2013). "Decision-tree early warning score (DTEWS) validates the design of the National Early Warning Score (NEWS)". Resuscitation. Elsevier Ireland.

Brady, P. W. et al. (2013). "Improving Situation Awareness to Reduce Unrecognized Clinical Deterioration and Serious Safety Events". Pediatrics. 131;e298 DOI: 10.1542/peds.2012-1364.

Burbey I. and Martin T. (2012). "A survey on predicting personal mobility”. International Journal of Pervasive Computing and Communications. Emerald.

Costa, C. A, Yamin, A. Geyer, C. (2008). "Towards a General Software Infrastructure for Ubiquitous Computing". IEEE Pervasive Computing. V. 7, p. 64-73.

Elgazzar K., Aboelfotoh M., Martin P. (2012). "Ubiquitous Health Monitoring Using Mobile Web Service”. Procedia Computer Science. V. 10, n. 0, p. 332 - 339.

Gelogo, Y. E., and Kim, H. K. (2013). "Unified Ubiquitous Healthcare System Architecture with Collaborative Model”. International Journal of Multimedia and Ubiquitous Engineering, 8(3). 
Gonçalves P. et al. (2012). "Reduced frequency of cardiopulmonary arrests by rapid response teams". Einstein. SciELO.

Hendrich, A. L. (2007). "Predicting Patient Falls". American Journal of Nursing. 107(11): 50-58.

Kirkland, L. et al. (2013). “A Clinical Deterioration Prediction Tool for Internal Medicine Patients". American Journal of Medical Quality. 28(2) 135-142.

Kitchenham, B. (2004). "Procedures for Performing Systematic Reviews". Joint Technical Report. Keele University.

Kyriacos, U. et al. (2014). "Monitoring Vital Signs: Development of a Modified Early Warning Scoring (Mews) System for General Wards in a Developing Country". PLoS ONE. 9(1): e87073. doi:10.1371/journal.pone.0087073

Konig I., Voigtmann C., Klein B., David K. (2011). "Enhancing alignment based context prediction by using multiple context sources: experiment and analysis". In Modeling and Using Context. Springer-Verlag, p. 159-172.

McCannon C., Hackbarth A., Griffin A. (2007). "Miles to Go: An Introduction to the 5 Million Lives Campaign". The Joint Commission Journal on Quality and Patient Safety, Volume 33, Number 8, pp. 477-484(8).

Morgan R. J. M, Williams F., Wright M. M. (1997). "An early warning scoring system for detecting developing critical illness". Clinical Intensive Care. 8: 100.

Offner P., Heit J., Roberts R. (2007). "Implementation of a Rapid Response Team Decreases Cardiac Arrest Outside of the Intensive Care Unit". Journal of TraumaInjury Infection \& Critical Care. OVID.

Patterson D., Liao L., Fox D., Kautz H. (2003). "Inferring High-Level Behavior from Low-Level Sensors". In Ubiquitous Computing, p. 73-89.

Rocha, C., Costa, C. A., Righi, R. R. (2015). Um modelo para monitoramento de sinais vitais do coração baseado em ciência da situação e computação ubíqua. SBCUP 2015.

Rosa J. H., Barbosa J. L.V., Ribeiro G. D. (2015). "ORACON: An Adaptive Model for Contexts Prediction”. Expert Systems With Applications, Elsevier.

Satyanarayanan M. (2001). "Pervasive computing: vision and challenges". IEEE Personal Communications. IEEE, 2001.

Sigg S. (2008). "Development of a Novel Context Prediction Algorithm and Analysis of Context Prediction Schemes". Kassel University Press.

Watcher R., Pronovost P. (2006). "The 100,000 Lives Campaign: A Scientific and Policy Review". Journal on Quality and Patient Safety.

Weiser M. (1991). "The computer for the twenty-first century". ACM SIGMOBILE Mobile Computing and Communications Review - Special issue dedicated to Mark Weiser. ACM Digital Library.

Weiss G., Hirsh H. (1998). "Learning to Predict Rare Events in Categorical Time-Series Data". In Proceedings of the Fourth International Conference on Knowledge Discovery and Data Mining, AAAI Press, Menlo Park, CA. 